\title{
Surface wave analysis with beamforming
}

\author{
Toshiro Tanimoto and Kenton Prindle \\ Institute for Crustal Studies and Department of Earth Science, University of California, Santa Barbara, California 93106, USA
}

(Received January 29, 2007; Revised March 27, 2007; Accepted April 2, 2007; Online published June 8, 2007)

\begin{abstract}
It is well known that off-great-circle path propagation causes a technical difficulty for surface wave analysis in higher frequency ranges. We propose a new approach that combines a beamforming technique and two-station phase velocity measurement to resolve this problem. Beamforming allows us to determine the correct azimuth of incoming surface waves which can be taken into account in phase velocity measurement. Beamforming results also support that a plane-wave approximation is mostly acceptable for frequencies up to about $50-60 \mathrm{mHz}$ (millihertz), although evidence of multipathing is occasionally recognized in beamforming results as multiple peaks. Application of this correction scheme for Rayleigh-wave data in Southern California seems to make the largest impact on the results of azimuthal anisotropy. Effects are not large for frequencies up to $30 \mathrm{mHz}$ but fast velocity axes in azimuthal anisotropy maps change significantly for higher frequencies.
\end{abstract}

Key words: Surface wave, wave propagation, earth structure.

\section{Introduction}

One of the most difficult problems in the analysis of teleseismic surface waves has been the so-called off-greatcircle path propagation for high frequency surface waves, noted at least since McGarr (1969a, b). On a global scale, long period surface waves mostly propagate along great circle paths but they start to deviate from great circle paths at periods about 50-60 seconds (e.g., Laske and Masters, 1996). This period range, however, may vary depending on geographical locations. If a region is near a sharp structural boundary, such as the ocean-continent boundary (thus both Japan and California), even longer-period waves may be susceptible to lateral refraction of surface waves.

Various groups are trying to resolve this type of problem in different ways; one can now take a completely numerical approach because of fast computers (e.g., Tape et al., 2006; Zhao and Jordan, 2006). Alternatively, one can take a more observational, wavefield modelling approach for an array (Friedrich et al., 1994; Pollitz, 1999; Bruneton et al., 2001; Yang and Forsyth, 2006). In this paper, we will take the latter approach and propose an approach that combines the beamforming method and two-station phase velocity measurement. Both the beamforming and phase velocity measurements are not new but their combination does not seem to have been attempted extensively, although Baumont et al. (2002) and Bourova et al. (2006) tried a somewhat similar approach to with a limited number of stations ( 3 or more). Essentially, what we are proposing is an improvement of such approaches using a seismic array.

Although Prindle and Tanimoto (2006) and Yang and Forsyth (2006) already published some results in Southern California, results for azimuthal anisotropy are incom-

Copyright (c) The Society of Geomagnetism and Earth, Planetary and Space Sciences (SGEPSS); The Seismological Society of Japan; The Volcanological Society of Japan; The Geodetic Society of Japan; The Japanese Society for Planetary Sciences; TERRAPUB. plete; Prindle and Tanimoto (2006) only discussed heterogeneity and Yang and Forsyth (2006) never really showed a map of azimuthal anisotropy; they only showed variations of azimuthal anisotropy with longitude that seem to suggest consistency with S-wave splitting data. Our analysis, with the use of the technique described in this paper (also in Prindle, 2006), seems to indicate that patterns of azimuthal anisotropy differ greatly from S-wave splitting results. Since anisotropy is important for understanding the tectonics in the region, clarification of the correct azimuthal anisotropy patterns is called for.

We apply the method to data from California, but it can be used for other areas with seismic broadband networks as, for example, Japan.

\section{Motivation from Particle Motion Analysis}

Our motivation for beamforming analysis arose from our particle motion analysis of Rayleigh waves. Two panels in Fig. 1 show horizontal particle motions of Rayleigh waves that arrived from the northwest quadrant (top panel) and also from the southwest quadrant (bottom); directions of horizontal particle motions are given by blue and those for great-circle paths are shown by red. These are the results at $0.04 \mathrm{~Hz}$

The top panel (Fig. 1) shows that blue lines are systematically oriented toward east-west in comparison to the greatcircle directions. On the other hand, the bottom panel shows generally good match between the great-circle directions and the observed particle motions. Clearly, the great-circle approximation is justified for the event in the bottom panel. This is generally true for events in the southwest azimuth in Southern California. This is not the case for the events in the northwest quadrant and some corrections for the deviation is definitely required in the analysis of this data set.

Another important feature in Fig. 1 is the fact that a plane-wave approximation may be a reasonable assump- 

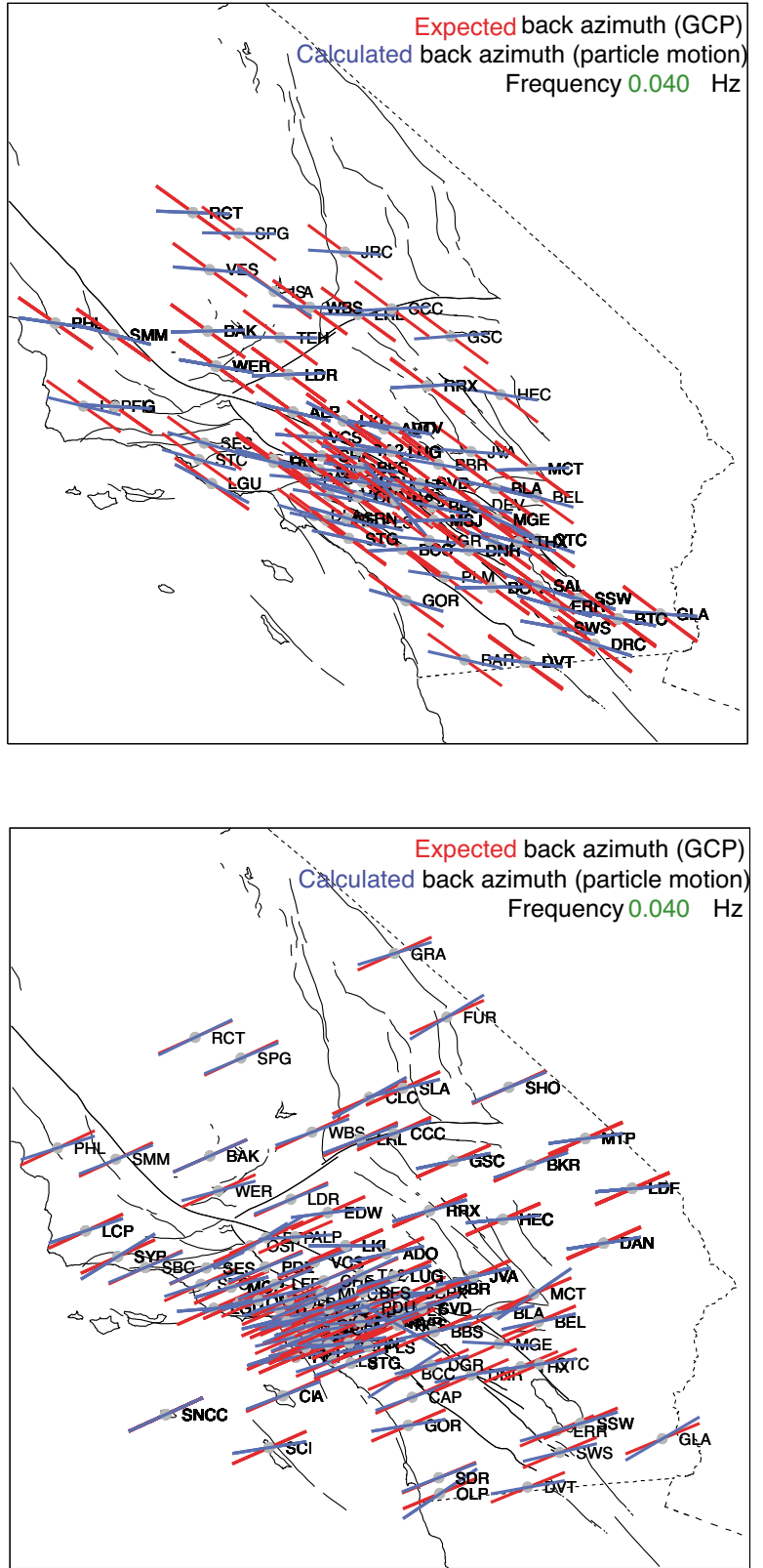

Fig. 1. (top) Comparison of great-circle directions (red) and the long axes of Rayleigh wave particle motions (blue). Blue lines are systematically shifted to east-west directions. (bottom) Rayleigh waves arrived from west for this event. Particle motions mostly match the great-circle direction.

tion at $0.04 \mathrm{~Hz}$, at least to first order. Directions of blue lines deviate from great-circle paths, especially in the top panel, but these observed directions are aligned parallel to each other mostly, suggesting a plane-wave like propagation. Some minor regional variations are seen which violate this plane-wave assumption but the most important first-order correction seems to be the correction for arrival azimuth of Rayleigh waves.

\section{Beamforming}

In order to determine the azimuth of arrival automatically, we adopted a beamforming approach. In this paper, we applied the beamforming to vertical component seismp- graphs for the entire array in Southern California. This study is thus limited to Rayleigh waves.

We used a simple stacking method (Aki and Richards, 1980; Johnson and Dudgeon, 1993), referenced to Pasadena station. Examples of beamforming results for frequencies between 0.01 and $0.06 \mathrm{~Hz}$ are shown in Figs. 2(a) and (b). The great-circle direction is shown by solid (black) line. Four concentric circles in each panel indicate the slowness of $0.1,0.2,0.3$ and $0.4(\mathrm{~s} / \mathrm{km})$ respectively.

Processing was done in the following way; Fourier spectra for the portion of time series that contain Rayleig waves were obtained first; they were obtained by using a cosine tapered filter. We made sure that results were not influenced strongly by a specific choice of detailed tapering range. We then used a frequency-domain beamforming approach, using the Fourier spectra from each station. We referenced all spectra to Pasadena (PAS) and stacked.

A case of Rayleigh wave arrival in the northwest quadrant is shown in Fig. 2(a). The maximum peak is indicated by red and blue denotes values that are $1 \mathrm{~dB}$ below the peak value. Denoting the peak location (red) as $\left(s_{x}, s_{y}\right)$ (in Figs. 2(a) and 2(b)), we can estimate phase velocity by $\left(1 / \sqrt{s_{x}^{2}+s_{y}^{2}}\right)$ and also its azimuth (thus deviations from great-circle directions).

A relatively broad but single peak is seen up to about $50 \mathrm{mHz}$ in Fig. 2(a). It is thus possible to determine the azimuth of arrival from the peak in the beamforming results. Note that locations of the peak start to deviate from the great-circle direction at frequencies about $15-20 \mathrm{mHz}$. Deviations in this figure are systematically to the west with respect to the great-circle directions, if one viewed the incoming waves at seismic stations. This is consistent with the particle-motion results in Fig. 1. Quantitatively, deviation angles from particle motion are as large as 30-40 degrees but vary from station to station to some extent. It is similar to variations in arrival azimuth estimates, shown later in Fig. 3(b), in which the average angle is about 20 degrees but a large number of data exist between 10 and 30 degrees.

Multiple peaks, or the evidence for multipathing, are obvious in results for $55 \mathrm{mHz}$ and $57.5 \mathrm{mHz}$. There are at least three peaks in each panel indicating three different wave packets from different azimuths. The proximity of peaks to the concentric circle for $0.3 \mathrm{~s} / \mathrm{km}$ suggests that they are all fundamental-mode Rayleigh waves, arriving from multiple azimuths.

Figure 2(b) shows the case of Rayleigh-wave arrival from the east. They are generally consistent with the greatcircle propagation but some panels, especially the ones at $37.5 \mathrm{mHz}, 42.5 \mathrm{mHz}$ and $47.5 \mathrm{mHz}$, show multiple peaks. The fact that these complications arise for intermediate frequency ranges indicate that multipathing problem is very hard to solve, even for frequencies below $50-60 \mathrm{mHz}$.

We created plots like Figs. 2(a) and (b) for 201 earthquakes in various azimuths. The common characteristic features in those results can be summarized in the following four points: (1) Relatively clean arrival angles can be determined for teleseismic Rayleigh waves for frequencies between 10 and $60 \mathrm{mHz}$. (2) At low frequency end, about $10 \mathrm{mHz}$, the peaks lie mostly on the great circle directions 
(a) $2003 / 5 / 26 / 9$ Lat 34.148 Lon 241.829 Azm -52.081

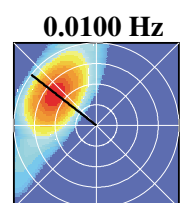

$0.0200 \mathrm{~Hz}$

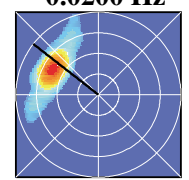

$0.0300 \mathrm{~Hz}$

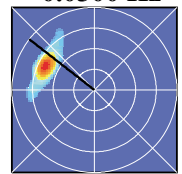

$0.0400 \mathrm{~Hz}$

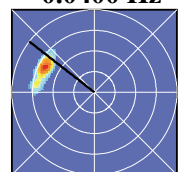

$0.0500 \mathrm{~Hz}$

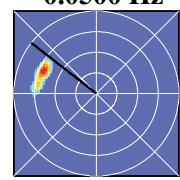

$0.0125 \mathrm{~Hz}$

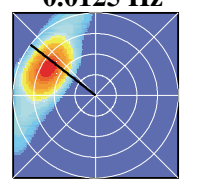

$0.0225 \mathrm{~Hz}$

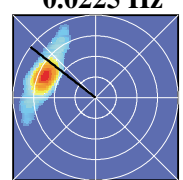

$0.0325 \mathrm{~Hz}$

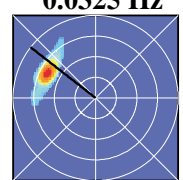

$0.0425 \mathrm{~Hz}$

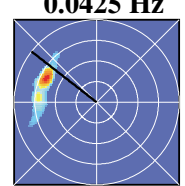

$0.0525 \mathrm{~Hz}$

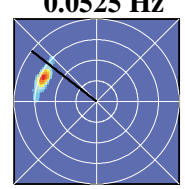

$0.0150 \mathrm{~Hz}$

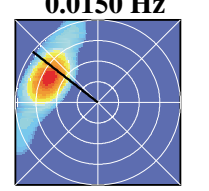

$0.0250 \mathrm{~Hz}$

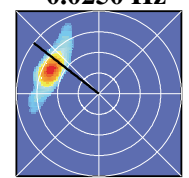

$0.0350 \mathrm{~Hz}$

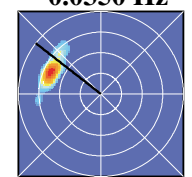

$0.0450 \mathrm{~Hz}$

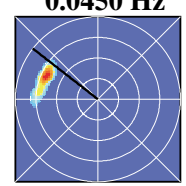

$0.0550 \mathrm{~Hz}$

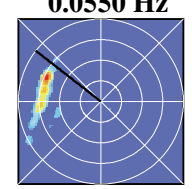

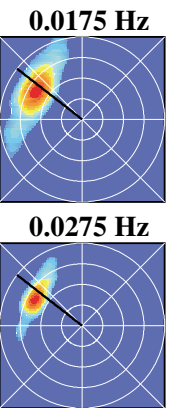

$0.0375 \mathrm{~Hz}$

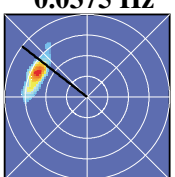

$0.0475 \mathrm{~Hz}$

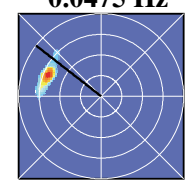

$0.0575 \mathrm{~Hz}$

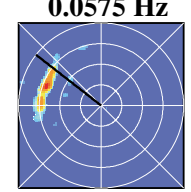

(b)

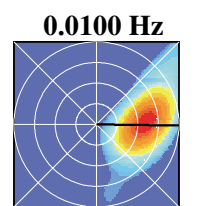

$0.0200 \mathrm{~Hz}$

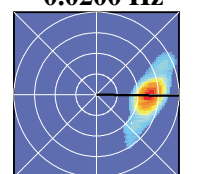

$0.0300 \mathrm{~Hz}$

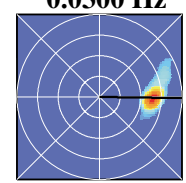

$0.0400 \mathrm{~Hz}$

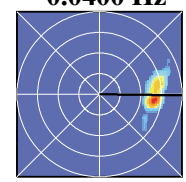

$0.0500 \mathrm{~Hz}$

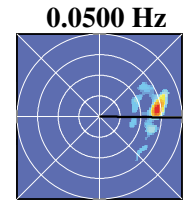

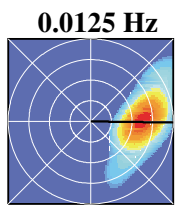

$0.0225 \mathrm{~Hz}$

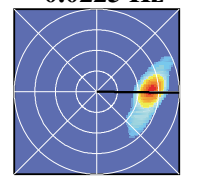

$0.0325 \mathrm{~Hz}$

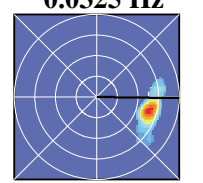

$0.0425 \mathrm{~Hz}$

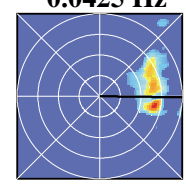

$0.0525 \mathrm{~Hz}$

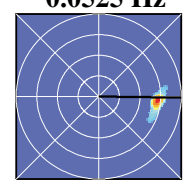

$0.0150 \mathrm{~Hz}$

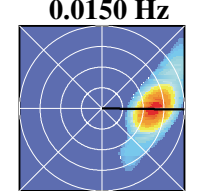

$0.0250 \mathrm{~Hz}$

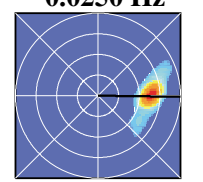

$0.0350 \mathrm{~Hz}$

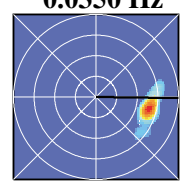

$0.0450 \mathrm{~Hz}$

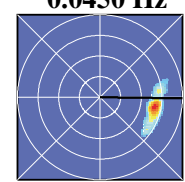

$0.0550 \mathrm{~Hz}$

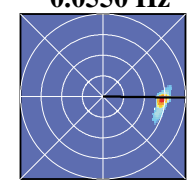

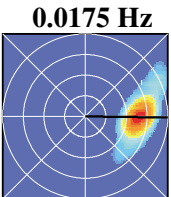

$0.0275 \mathrm{~Hz}$

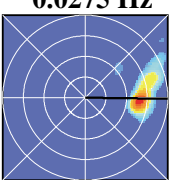

$0.0375 \mathrm{~Hz}$

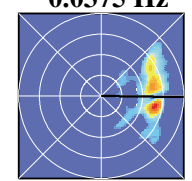

$0.0475 \mathrm{~Hz}$

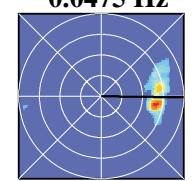

$0.0575 \mathrm{~Hz}$

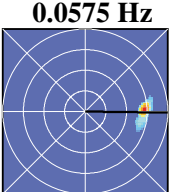

Fig. 2. (a) Beamforming results from $0.01 \mathrm{~Hz}$ to $0.0575 \mathrm{~Hz}$ for Rayleigh waves from the northwest quadrant. The peak value is denoted by red. Intermediate amplitudes are in yellow and small ampliudes are in blue. Great-circle directions are indicated by black lines. The peak is broad at 0.01 $\mathrm{Hz}$ because size of the array is small for this frequency. The peaks (red) are typically shifted to the west of black lines. Clear multipathing is seen in results at $55 \mathrm{mHz}$ and $57.5 \mathrm{mHz}$. (b) Beamforming results for arrival from the east. The peak locations are mostly on the great-circle direction. Multipathing is seen in results at $37.5 \mathrm{mHz}, 42.5 \mathrm{mHz}$ and $47.5 \mathrm{mHz}$, however.

but the resolution is not good (the width of peaks is broad) due to the size of an seismic array. (3) Systematic deviations from great circle directions are seen in higher frequencies, in some cases starting at about $15 \mathrm{mHz}$, but typically at about $30 \mathrm{mHz}$. In most case, a single arrival azimuth can be determined. (4) However, multipathing is occasionally seen for intermediate frequencies (between 10 and $60 \mathrm{mHz}$ ) and its occurrence is hard to predict. We have not found any systematics in the emergence of these multipathing effects.

\section{Proposed approach}

We made plots for angles of deviation from the greatcircle paths for 201 events. The angles were plotted as a function of frequency for different azimuth windows. Measured angles (deviations) show fair amount of scatter from event to event as two top panels in Figs. 3(a) and (b) show. These scatters might cause some problems in the analysis, if we applied the beamforming method on an event-by-event basis. On the other hand, there are clear systematics in the distributions of measured angles in both figures. We thus decided to estimate the arrival azimuth for a given azimuth window by computing the means and errors for these points.

After trials and errors, we decided to divide the entire 360-degree range in five different azimuth windows. They are $0-110,110-180,180-240,240-300$ and 300-360 degrees clockwise from North.

Figure 3(a) shows the results for azimuth between 180 and 240 degrees (southwest). There are many earthquakes in this range because this range includes earthquakes in the South Pacific. Deviations from the great-circle paths are generally small in this azimuth.

Figure 3(b), for the azimuth between 300 and 360 degrees, shows the largest deviation from the great circle directions among the five azimuth windows. This azimuth range includes earthquakes from Alaska, Kuril, Japan and Taiwan. Rayleigh waves in this azimuth window tend to propagate at grazing angles along sharp structural contrasts in subduction zones (Kuril, Aleutian) and the continentocean boundaries off the coast of western United States. For this azimuth range, deviations are seen at frequencies as low as $15 \mathrm{mHz}$ and seem to grow for higher frequencies and reach 20 degrees at $50 \mathrm{mHz}$.

Computed means and error bars (one sigma) are given in the bottom panels (Figs. 3(a) and (b)). Summary of results for all five azimuth windows are shown in Fig. 3(c).

As a first step, we applied the results given in Fig. 3(c). Since the plane-wave approximation seems to be acceptable, we applied corrections to the azimuth of incoming waves and measured phase velocities between pairs of stations as in our previous work (e.g., Tanimoto and Prindle, 2002). Detailed analysis can be found in Prindle (2006).

Strictly speaking, deviation angles should also be a function of epicentral distance and azimuth in a threedimensional structure (Cotte et al., 2000). Our proposed 
(a) Azimuth between 180 and 240

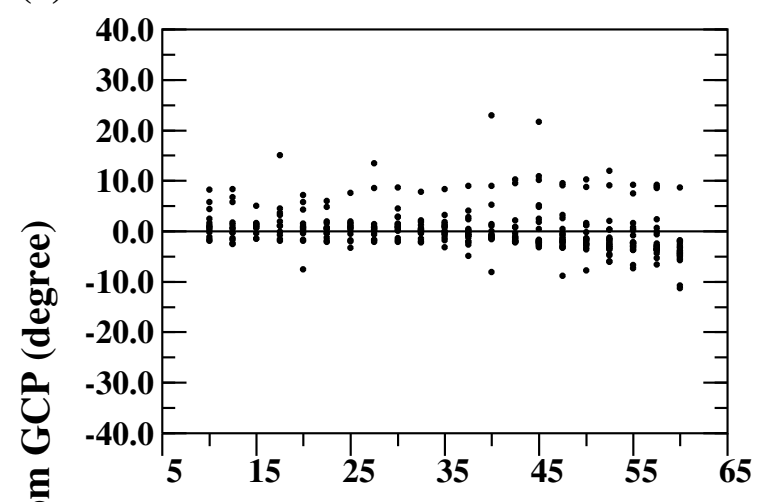

(b)
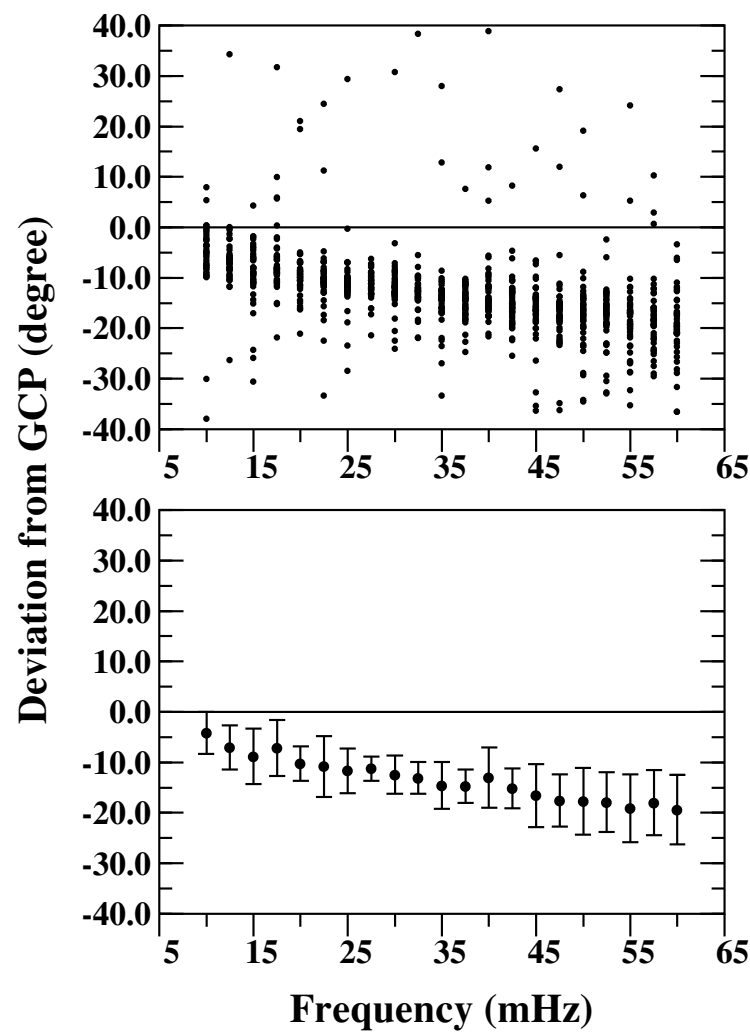

Fig. 3. The data were divided into five azimuth windows. For each window, angles of deviation from great-circle direction as a function of frequency are plotted. Raw data are given at top and the means and standard errors are shown at bottom. Results from two windows are shown 180-240 degrees (a) and 300-360 degrees (b). (c) Summary of angle-deviations for the five windows. (c)
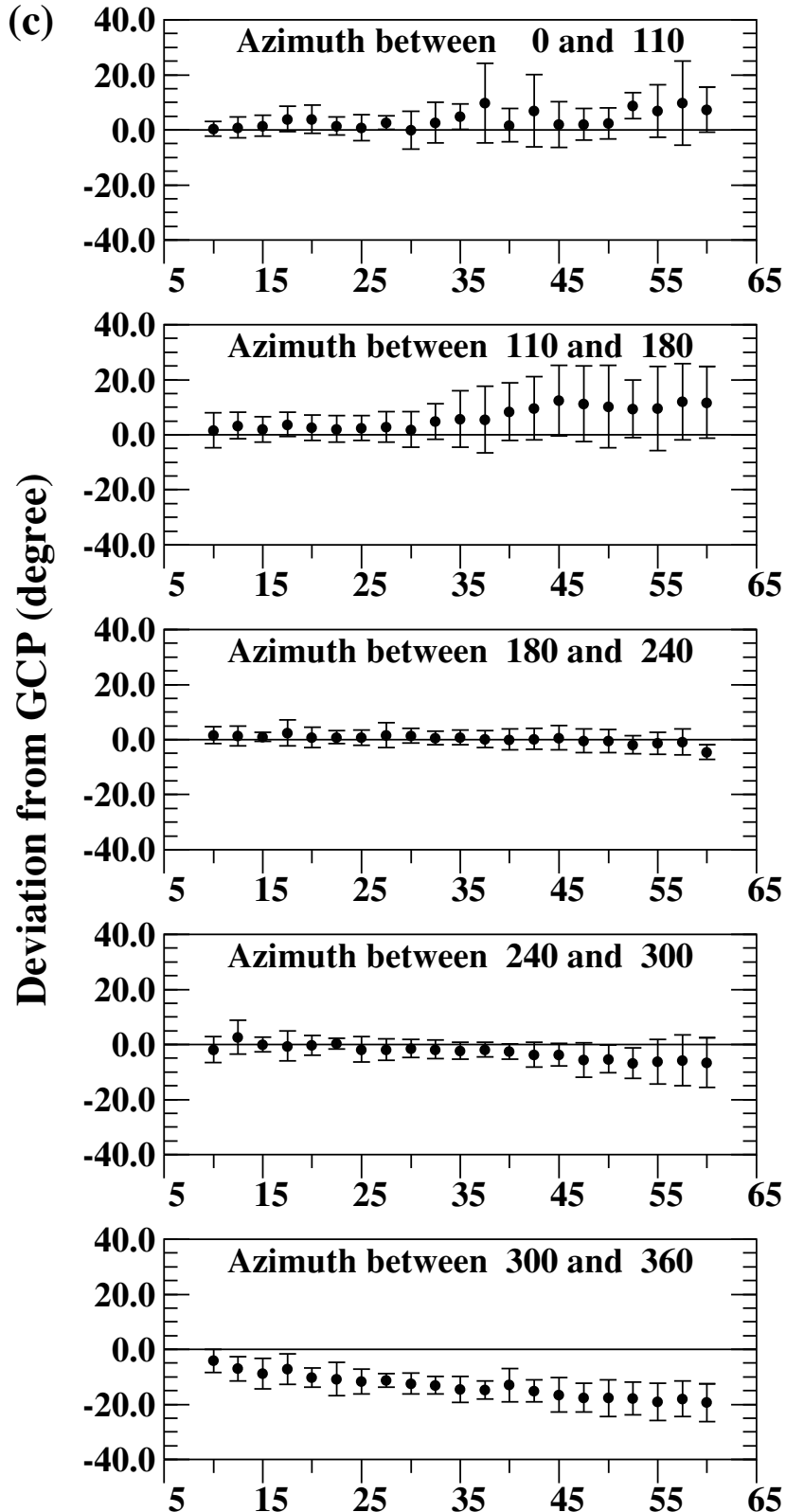

Frequency $(\mathrm{mHz})$

Fig. 3. (continued).

scheme is based on empirical evidence that azimuth alone can be used to predict deviations angles. This situation occurred probably because there are only one seismically active region within a given azimuth. Therefore, this empirical approach is not guaranteed to work for other regions.

\section{Impact on azimuthal anisotropy}

We re-inverted phase velocity data using the proposed approach and compared with the results obtained by us previously (Prindle and Tanimoto, 2006). We found little difference in the patterns of S-wave velocity variations (heterogeneity) but substantial differences in azimuthal anisotropy maps. We briefly describe our results on anisotropy below. Our basic approach is similar to Tanimoto and Anderson (1985) and we only kept up to $2 \theta$ azimuthal dependence. 

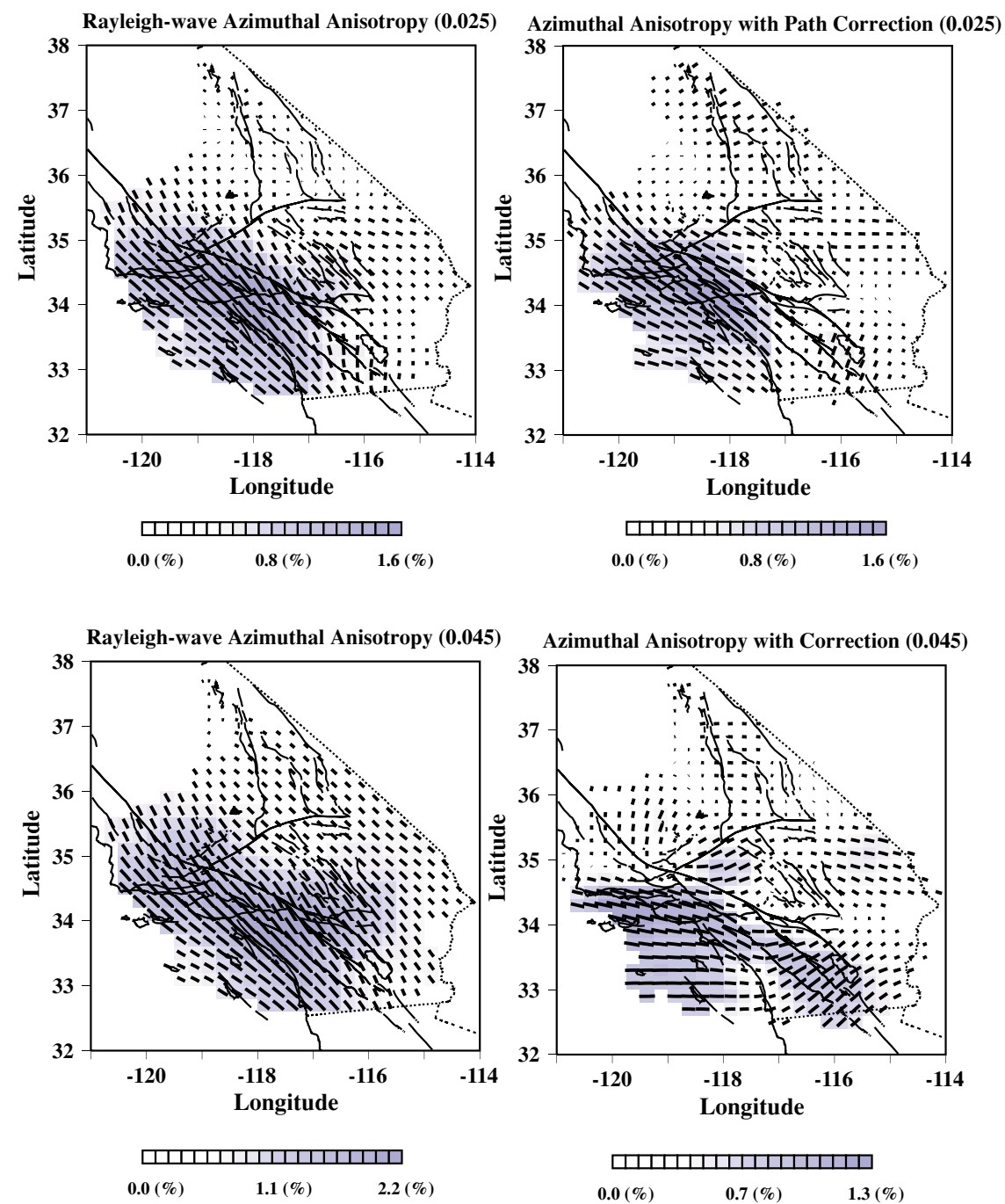

Fig. 4. Phase velocities were measured by taking into account the five-window scheme in Fig. 3(c). The most striking changes are seen in azimuthal anisotropy results at high frequencies. The left column shows the results without the correction ( $25 \mathrm{mHz}$ at top and $45 \mathrm{mHz}$ at bottom) and the right columns show results with the correction. While the changes in the results for $25 \mathrm{mHz}$ are not large, directions of the fast velocity in the $45 \mathrm{mHz}$ map changes dramatically. They illustrate the importance of taking into account the correct arrival azimuths in phase velocity measurements.

We solved for homogeneous block structure using ray theory.

Figure 4 shows our results for azimuthal anisotropy at two frequencies, 25 and $45 \mathrm{mHz}$, with and without the correction procedure we propose above. The left panels are the results without the correction (thus the great-circle path propagation) and the right panels are the results with the correction, using the five different azimuth range into consideration (Fig. 3(c)).

The results at $25 \mathrm{mHz}$, given at top, do not show major differences due to the incorporation of the beamforming results. However, at $45 \mathrm{mHz}$, the directions of fastest velocity azimuth change rather dramatically. On the west side of the San Andreas fault, the fastest directions are mostly northwest-southeast directions without our proposed correction, but they turn out to be almost east-west after the correction. There are fewer stations off the coast, but islands are mostly occupied by stations, thereby giving us some resolving power even in this area. Because deviations from the great-circle paths are much larger at $45 \mathrm{mHz}$ than $25 \mathrm{mHz}$, this result is most likely related to the corrections we apply to data. It may even imply that the correct directions of azimuthal anisotropy cannot be recovered unless the correct arrival azimuth is taken into account in the phase velocity analysis.

This result may not be surprising since a larger deviation from a great-circle path tend to lead to a larger velocity estimate (Pedersen, 2006). So the directions of strong great-circle deviation may tend to become a fast propagation direction.

\section{Conclusion}

We proposed a new Rayleigh wave analysis technique that combines the beamforming of array data (for Southern California Network) and two-station phase velocity measurement.

The beamforming technique allows us to determine the angles of deviations as a function of frequency. In some azimuth windows, this deviation is large and correction due to this deviation must be applied in phase/group velocity measurement. We also noted from particle motion analysis that, as long as this incoming azimuth correction is applied, 
a plane-wave assumption seems to hold (to first order).

We developed a specific scheme of dividing the whole azimuth into five different azimuth windows for data in Southern California. The procedure makes a large impact on the results of azimuthal anisotropy, especially for higher frequencies above $30 \mathrm{mHz}$. Since the directions of the fast velocity axes is perhaps one of the most important aspects of anisotropic structure for tectonic interpretations, correcting for the angles of deviations from great-cirle paths seems critical for surface wave analysis.

Acknowledgments. This study was supported by the Southern California Earthquake Center (SCEC). SCEC is funded by NSF cooperative Agreement EAR-0106924 and USGS Cooperative Agreement 02HQAG0008. The SCEC contribution number for this paper is 1037 .

\section{References}

Aki, K. and P. G. Richards, Quantitative Seismology, W. H. Freeman and Company, San Francisco, 1980.

Baumont, D., A. Paul, G. Zandt, S. Beck, and H. Pedersen, Lithospheric structure of the central Andes based on surface wave dispersion, $J$. Geophys. Res., 107, B12, 2371, doi:10.1029/2001JB000345, 2002.

Bourova, E., I. Kassaras, H. A. Pedersen, T. Yanovskaya, and D. Hatzfeld, Constraints o absolute S velocities beneath the Aegean Sea from surface wave analysis, Geophys. J. Int., 160, 1006-1019, doi:10.1111/j.1365246X.2005.02565.x, 2005.

Bruneton, M., V. Farra, H. A. Pedersen, and teh SVEKALAPKO Seismic Tomography Working Group, Non-linear surface wave phase velocity inversion based on ray theory, Geophys. J. Int., 151, 583-596, 2002.

Cotte, N., H. A. Pedersen, M. Campillo, V. Farra, and Y. Cansi, Off-greatcircle propagation of intermediate-period surface waves observed on a dense array in the French Alps, Geophys. J. Int., 142, 825-840, 2000.

Friedrich, W., E. Wielandt, and S. Strange, Non-plane geometries of seismic surface wavefields and their implications for regional surface wave tomography, Geophys. J. Int., 119, 931-948, 1994.
Johnson, Don H. and D. E. Dudgeon, Array Signal Processing, P T R Prentice-Hall Inc., Upper Saddle River, New Jersey, 1993.

Laske, G. and G. Masters, Constraints on global phase velocity maps by long-period polarization data, J. Geophys. Res., 101, B7, 16059-16075, 1996.

McGarr, A., Amplitude variations of Rayleigh waves-Across a continental margin, Bull. Seism. Soc. Am., 59, 1281-1305, 1969a.

McGarr, A., Amplitude variations of Rayleigh waves-Horizontal refraction, Bull. Seism. Soc. Am., 59, 1307-1334, 1969b.

Pedersen, H. A., Impacts of non-plane waves on two-station measurements of phase velocities, Geophys. J. Int., 165, 279-287, doi:10.1111/j.1365246X.2006.02893.x, 2006.

Pollitz, F., Regional velocity structure in nortehrn California from inversion of scattered seismic surface waves, J. Geophys. Res., 104, B7, 15043-15072, 1999.

Prindle, K., Souther California Surface Wave Analysis: Recovery of Three-dimensional S-wave Velocity Structure, the Finite Frequency, Off Great-Circle Propagation and Azimuthal Anisotropy, Ph.D. thesis, University of California, Santa Barbara, 2006.

Prindle, K. and T. Tanimoto, Teleseismic Surface Wave study for S-wave velocity structure under an array: Southern California, Geophys. J. Int., 166, 601-621, 2006.

Tanimoto, T. and Don L. Anderson, Lateral Heterogeneity and Azimuthal Anisotropy of the Upper Mantle: Love and Rayleigh Waves 100-250 s, J. Geophys. Res., 90, 842-1858, 1985.

Tanimoto, T. and K. Prindle, Three-dimensional S-wave velocity structure in Southern California, Geophys. Res. Lett., 29, 8, 1-4, doi:1029/ 2001GL013486, 2002.

Tape, C., Q. Liu, and J. Tromp, Finite-frequency tomography using adjoint methods-Methodology and examples using membrane surface waves. Geophys. J. Int., 2006 (in press).

Yang, Y. and D. W. Forsyth, Rayleigh wave phase velocitiers, small-scale convection, and azimuthal anisotropy beneath souther California, $J$. Geophys. Res., 111, B07306, doi:10.1029/2005JB004180, 2006.

Zhao, L. and T. Jordan, STructural sensitivity of finite-frequency seismic waves: A full-wave approach, Geophys. J. Int., 165, 981-990, 2006.

T. Tanimoto (e-mail: toshiro@geol.ucsb.edu) and K. Prindle 